\title{
A note about special cycles on moduli spaces of K3 surfaces
}

\author{
Stephen Kudla
}

\begin{abstract}
We describe the application of the results of Kudla-Millson on the modularity of generating series for cohomology classes of special cycles to the case of lattice polarized K3 surfaces. In this case, the special cycles can be interpreted as higher Noether-Lefschetz loci. These generating series can be paired with the cohomology classes of complete subvarieties of the moduli space to give classical Siegel modular forms with higher Noether-Lefschetz numbers as Fourier coefficients. Examples of such complete families associated to quadratic spaces over totally real number fields are constructed.
\end{abstract}

\section{Introduction}

This article contains a short survey of some results about special cycles on certain Shimura varieties that occur as moduli spaces of lattice polarized K3 surfaces. The two points that may be of interest are the Siegel modular forms arising as generating series for higher Noether-Lefschetz numbers and the description of some complete subvarieties in certain of these moduli spaces. The families of K3 surfaces parametrized by these subvarieties ought to have particularly nice properties and I do not know to what extent they already occur implicitly or explicitly in the literature. Finally, I ask the indulgence of the experts in this area for my very naive treatment of things that may be very well known to them.

Stephen Kudla Department of Mathematics, University of Toronto, 40 St George St. BA6290, Toronto, ON M5S 2E4, Canada e-mail: skudla@math.toronto.edu 


\section{Special cycles for orthogonal groups}

We begin by reviewing a very special case of old joint work with John Millson, [14], 15], 16].

\section{1}

Let $L,($,$) be a lattice with a \mathbb{Z}$-valued symmetric bilinear form of signature $(2, n)$. In particular, for $V=L_{\mathbb{Q}}=L \otimes_{\mathbb{Z}} \mathbb{Q}$, the dual lattice

$$
L^{\vee}=\{x \in V \mid(x, L) \subset \mathbb{Z}\}
$$

contains $L$. Let

$$
\begin{aligned}
D=D(L) & =\left\{w \in V_{\mathbb{C}} \mid(w, w)=0,(w, \bar{w})>0\right\} / \mathbb{C}^{\times} \\
& \simeq\left\{\text { oriented positive 2-planes in } V_{\mathbb{R}}\right\} \\
& \simeq \mathrm{SO}\left(V_{\mathbb{R}}\right) / K
\end{aligned}
$$

be the associated symmetric space, where $K \simeq S O(2) \times S O(n)$ is the stabilizer of an oriented positive 2-plane. Here $V_{\mathbb{C}}=V \otimes_{\mathbb{Q}} \mathbb{C}\left(\right.$ resp. $\left.V_{\mathbb{R}}=V \otimes_{\mathbb{Q}} \mathbb{R}\right)$, and we sometimes write $\underline{w}$ for the image of $w \in V_{\mathbb{C}}$ in $D$. Let $\Gamma_{L} \subset \mathrm{SO}(V)$ be the isometry group of $L$ and let $\Gamma \subset \Gamma_{L}$ be a subgroup of finite index. Then

$$
M_{\Gamma}=\Gamma \backslash D(L), \quad \operatorname{dim}_{\mathbb{C}} M_{\Gamma}=n
$$

is (isomorphic to) a quasi-projective variety. This variety is a connected component of a Shimura variety 1 and has a model defined over a cyclotomic field. For small values of $n$, this space can be the moduli space of polarized K3 surfaces and, for smaller values, of abelian varieties.

\section{2}

To define special cycles, suppose that $x \in L$ is a vector with $(x, x)<0$, and let

$$
D_{x}=\{\underline{w} \in D \mid(x, \underline{w})=0\}=D\left(L \cap x^{\perp}\right) .
$$

Thus $D_{x}$ has codimension 1 in $D$ and gives rise to a divisor

$$
Z(x): \Gamma_{x} \backslash D_{x} \longrightarrow \Gamma \backslash D=M_{\Gamma},
$$

\footnotetext{
${ }^{1}$ For $n \leq 2$, we need to add the condition that $\Gamma$ be a congruence subgroup; this is
} automatic for $n \geq 3$. In general, $M_{\Gamma}$ can have 2 components, since $D(L)$ does. 
in $M_{\Gamma}$, where $\Gamma_{x}$ is the stabilizer of $x$ in $\Gamma$. We call such a divisor, which depends only on the $\Gamma$-orbit of $x$, a special divisor.

Composite divisors are defined as follows. Let $\Gamma_{L}^{o} \subset \Gamma_{L}$ be the subgroup of isometries that act trivially on $L^{\vee} / L$, and suppose that $\Gamma \subset \Gamma_{L}^{o}$. For $t \in \mathbb{Z}_{>0}$ and $h \in L^{\vee}$ a coset representative for $L^{\vee} / L$, let

$$
L(t, h)=\{x \in L+h \mid(x, x)=-t\} .
$$

There is an associated special divisor

$$
Z(t, h)=\sum_{\substack{x \in L(t, h) \\ \bmod \Gamma}} Z(x) .
$$

Remark. For the following observation, see [33], Lemma 1.7. The space $D$ parametrizes polarized Hodge structures of weight 2 on the rational vector space $V$ with $\operatorname{dim}_{\mathbb{C}} V_{\mathbb{C}}^{2,0}=1$. Such a HS is simple if it does not contain any proper rational Hodge substructure. Then, in fact, the polarized HS corresponding to $\underline{w} \in D$ is simple if and only if $\underline{w} \notin D_{x}$ for any nonzero vector $x \in V$. Thus the set

$$
D-\bigcup_{x \in V, x \neq 0} D_{x}
$$

parametrizes the simple HS's of this type.

More generally, for $1 \leq r \leq n$, consider an $r$-tuple of vectors

$$
\mathbf{x}=\left[x_{1}, \ldots, x_{r}\right], \quad x_{i} \in V,
$$

and suppose that

$$
T(\mathbf{x})=-(\mathbf{x}, \mathbf{x})=-\left(\left(x_{i}, x_{j}\right)\right)>0 .
$$

Let

$$
\begin{aligned}
D_{\mathbf{x}} & =\{\underline{w} \in D \mid(\mathbf{x}, \underline{w})=0\}, \\
\Gamma_{\mathbf{x}} & =\text { stablizer of } \mathbf{x} \text { in } \Gamma,
\end{aligned}
$$

and

$$
Z(\mathbf{x}): \Gamma_{\mathbf{x}} \backslash D_{\mathbf{x}} \longrightarrow \Gamma \backslash D=M_{\Gamma} .
$$

The condition $T(\mathbf{x})>0$ implies that $D(\mathbf{x})$ has codimension $r$ in $D$ so that the special cycle $Z(\mathbf{x})$ has codimension $r$ in $M_{\Gamma}$. Again, this cycle depends only on the $\Gamma$-orbit of $\mathbf{x}$.

Again there is a composite version. For

$$
T \in \operatorname{Sym}_{r}(\mathbb{Z})_{>0}, \quad \mathbf{h} \in\left(L^{\vee}\right)^{r},
$$

let 


$$
L(T, \mathbf{h})=\left\{\mathbf{x} \in L^{r}+\mathbf{h} \mid T(\mathbf{x})=T\right\},
$$

and define the cycle

$$
Z(T, \mathbf{h})=\sum_{\substack{\mathbf{x} \in L(T, \mathbf{h}) \\ \bmod \Gamma}} Z(\mathbf{x}) .
$$

These are the special cycles in question.

Remarks. a) In the rest of this note, we will suppress the coset parameter $h$, although it plays an evident and important role in many places in the literature.

b) We can make the same construction with any $T \in \operatorname{Sym}_{r}(\mathbb{Z})_{\geq 0}$ except that we require that for $\mathbf{x} \in L(T, \mathbf{h})$ the subspace spanned by the components of $\mathbf{x}$ has dimension equal to the rank of $T$. We write $Z^{\text {naive }}(T, \mathbf{h})$ for the resulting cycle. It has codimension equal to the rank of $T$. For example, for $\mathbf{h}=0$,

$$
Z^{\text {naive }}(0, \mathbf{h})=M_{\Gamma},
$$

while, for $\mathbf{h} \neq 0, Z^{\text {naive }}(0, \mathbf{h})$ is empty.

\section{Modular generating series}

First, for $\tau=u+i v \in \mathfrak{H}$, the upper half-plane, consider the series

$$
\phi_{1}(\tau)=[Z(0)]+\sum_{t \in \mathbb{Z}_{>0}}[Z(t)] q^{t}, \quad[Z(t)] \in H^{2}\left(M_{\Gamma}\right) .
$$

Here $[Z(0)]=[\omega]=c_{1}(\mathcal{L})$ is the Chern class of the tautological line bundle

$$
\mathcal{L} \longrightarrow M_{\Gamma}
$$

defined by:

$$
\mathcal{L}=b^{*}(\mathcal{O}(-1)), \quad b: D(L) \longrightarrow D(L)^{\vee}
$$

where

$$
D^{\vee}(L)=\left\{w \in V_{\mathbb{C}} \mid(w, w)=0\right\} / \mathbb{C}^{\times} \subset \mathbb{P}\left(V_{\mathbb{C}}\right)
$$

is the compact dual of $D(L)$. Here $H^{r}\left(M_{\Gamma}\right)$ is the usual Betti cohomology group of the (quasi-projective) variety $M_{\Gamma}$ with complex coefficient: 2 .

2 If $\Gamma$ has fixed points on $D(L), M_{\Gamma}$ is viewed as an orbifold and $H^{r}\left(M_{\Gamma}\right)$ is the space of $\Gamma / \Gamma_{1}$-invariants in $H^{r}\left(M_{\Gamma_{1}}\right)$ where $\Gamma_{1} \subset \Gamma$ is a normal subgroup of finite index which acts freely on $D(L)$. 
Theorem 2.1 (16]) $\phi_{1}(\tau)$ is an elliptic modular form of weight $\frac{n}{2}+1$ and level determined 3 by $L$, valued in $H^{2}\left(M_{\Gamma}\right)$.

The analogous generating series with values in the first Chow group $\mathrm{CH}^{1}\left(M_{\Gamma}\right) \otimes_{\mathbb{Z}} \mathbb{C}$ of $M_{\Gamma}$ was considered by Borcherd: 4

Theorem $2.2([2])$

$$
\phi_{1}^{C H}(\tau)=\{Z(0)\}+\sum_{t \in \mathbb{Z}_{>0}}\{Z(t)\} q^{t}, \quad\{Z(t)\} \in \mathrm{CH}^{1}\left(M_{\Gamma}\right) \otimes \mathbb{C}
$$

is an elliptic modular form of weight $\frac{n}{2}+1$, etc. Its image under the cycle class map

$$
\mathrm{CH}^{1}\left(M_{\Gamma}\right) \otimes \mathbb{C} \longrightarrow H^{2}\left(M_{\Gamma}\right)
$$

is $\phi_{1}(\tau)$.

More generally, for any $r, 1 \leq r \leq n$, and for $\tau=u+i v \in \mathfrak{H}_{r}$, the Siegel space of genus $r$, we can define a generating series

$$
\begin{aligned}
\phi_{r}(\tau) & =\sum_{\substack{T \in \operatorname{Sym}_{r}(\mathbb{Z}) \\
T \geq 0}}\left[Z^{\text {naive }}(T)\right] \cup[\omega]^{r-\operatorname{rank} T} q^{T}, \\
& =[\omega]^{r}+\sum_{\operatorname{rank} T<r}\left[Z^{\text {naive }}(T)\right] \cup[\omega]^{r-\operatorname{rank} T} q^{T}+\sum_{T \in \operatorname{Sym}_{r}(\mathbb{Z})_{>0}}[Z(T)] q^{T} .
\end{aligned}
$$

The point is that we have shifted the classes $\left[Z^{\text {naive }}(T)\right]$ by a suitable power of $[\omega]$ so that all of the coefficients lie in $H^{2 r}\left(M_{\Gamma}\right)$.

Theorem 2.3 ([16]) (contd.) $\phi_{r}(\tau)$ is a Siegel modular form of weight $\frac{n}{2}+1$ and level determined by $L$, valued in $H^{2 r}\left(M_{\Gamma}\right)$.

Remark 2.4 (1) In [11] I asked whether the Chow group version $\phi_{r}^{C H}(\tau)$ is a Siegel modular form valued in $\mathrm{CH}^{r}\left(M_{\Gamma}\right)$. Using Borcherds' result mentioned above and an inductive argument based on Fourier-Jacobi expansions, Wei Zhang proved this in his Columbia thesis, 34], conditionally on some finiteness/convergence assumption.

(2) A key point is that the pairing of $\phi_{r}(\tau)$ with any compactly supported class $c \in H_{c}^{2 n-2 r}\left(M_{\Gamma}\right)$ defines a classical Siegel modular form 5 of the same weight and level as $\phi_{r}(\tau)$.

3 This means that the components of $\phi_{1}(\tau)$ with respect to any basis of the finite dimensional space $H^{2}\left(M_{\Gamma}\right)$ are scalar valued modular forms of the given weight and level. The level divides $4\left|L^{\vee} / L\right|$ and is determined by the usual recipe for theta functions, cf., for example, [30], section 2.

4 More precisely, he views $M_{\Gamma}$ as an orbifold/stack and defines a group by generators and relations that maps to the usual Chow group, at least after tensoring with $\mathbb{C}$.

${ }^{5}$ This form will be vector-valued if we keep track of the parameter $\mathbf{h} \in L^{\vee} / L$. 
(3) It is worth noting that the modularity of the $\phi_{r}(\tau)$ is proved by constructing a theta function $\theta\left(\tau, \varphi_{K M}^{(r)}\right)$ valued in $A^{(r, r)}\left(M_{\Gamma}\right)$ the space of smooth differential forms of type $(r, r)$ on $M_{\Gamma}$. This theta function is a non-holomorphic modular form of weight $\frac{n}{2}+1$ in $\tau$, analogous to the classical Siegel theta function for an indefinite quadratic form. Moreover, it is a closed $(r, r)$-form and its cohomology class $\left[\theta\left(\tau, \varphi_{K M}^{(r)}\right)\right] \in H^{(r, r)}\left(M_{\Gamma}\right)$ is $\phi_{r}(\tau)$. In particular, if the class $c \in H_{c}^{2 n-2 r}\left(M_{\Gamma}\right)$ is the class of an algebraic cycle $c=[S]$, as will be the case in several examples below, the pairing of $c$ and $\phi_{r}(\tau)$ is given by an integral

$$
c \cdot \phi_{r}(\tau)=\int_{S} \theta\left(\tau, \varphi_{K M}^{(r)}\right) .
$$

(4) The extension of such integrals to classes c that are not compactly supported has been studied in important work of Funke and Millson, [5], [6], [7], and involves interesting correction terms related to the boundary of the Borel-Serre compactification of $M_{\Gamma}$.

\section{The case of K3 surfaces}

In some cases, the $M_{\Gamma}$ 's and the $Z(T)$ 's can be interpreted in terms of moduli spaces of lattice polarized 63 surfaces. Here is an amateur's version of this. Start from the K3 lattice:

$$
K=H^{3} \oplus E_{8}(-1)^{2}, \quad \operatorname{sig}(K)=(3,19),
$$

where $H$ is the unimodular hyperbolic plane, and, letting $K_{\mathbb{Q}}=K \otimes_{\mathbb{Z}} \mathbb{Q}$, choose an orthogonal decomposition

$$
K_{\mathbb{Q}}=V \oplus V^{\prime}
$$

with

$$
\operatorname{sig}\left(V^{\prime}\right)=(1,19-n), \quad \operatorname{sig}(V)=(2, n),
$$

for some $n, 0 \leq n \leq 19$. Let

$$
L=K \cap V, \quad L^{\prime}=K \cap V^{\prime},
$$

be the corresponding primitive sublattices in $K$. Note that they are both even integral.

A marked $L^{\prime}$-polarized $\mathrm{K} 3$ surface is a collection $(X, u, \lambda)$ where $X$ is an algebraic K3 surface over $\mathbb{C}$,

$$
u: H^{2}(X, \mathbb{Z}) \stackrel{\sim}{\longrightarrow} K
$$

${ }^{6}$ A good references for lattice polarized K3 surfaces are [3] and [4]. 
is an isometry for the intersection form on $H^{2}(X, \mathbb{Z})$, a marking, and

$$
\lambda: L^{\prime} \hookrightarrow \operatorname{Pic}(X) \subset H^{2}(X, \mathbb{Z})
$$

is an embedding such that

$$
u \circ \lambda: L^{\prime} \hookrightarrow K
$$

is the given inclusion. Moreover, $\lambda$ is required to satisfy the 'ample cone' condition], cf. section 10 of 4 .

These conditions imply that

(i) the embedding $\lambda: L^{\prime} \hookrightarrow \operatorname{Pic}(X)$ is primitive and isometric

(ii) the period point of $(X, u)$ lies in $D(V)$.

Recall that the period point of $(X, u)$ is the complex line $u_{\mathbb{C}}\left(H^{2,0}(X)\right)$, where $u_{\mathbb{C}}: H^{2}(X, \mathbb{Z}) \otimes_{\mathbb{Z}} \mathbb{C} \longrightarrow K_{\mathbb{C}}$ is the complex linear extension of $u$. The moduli space of such gadgets $(X, \lambda)$, obtained by eliminating the marking, is the quotient

$$
\begin{gathered}
M_{\Gamma}=\Gamma \backslash D(V), \\
\Gamma=\left\{\gamma \in \Gamma_{L}|\gamma|_{L^{\vee} / L}=1\right\} .
\end{gathered}
$$

This gives a moduli interpretation of $M_{\Gamma}$. Details of this construction can be found in [4], section 10 .

Remark 3.1 (1) It is easy to check that, for $n \leq 17$, any rational quadratic space of signature $(2, n)$ can occur as $V$. If $n=18$ or 19 , there are restrictions on $V$, since then $L_{\mathbb{Q}}^{\prime}$ has rank 2 or 1 .

(2) In certain cases, a precise description of what lattices $L^{\prime}$ 's and $L$ 's can occur was given by Nikulin [26]. As summarized in [25], section 2, the result is the following. If $0 \leq n \leq 9$, then any even integral lattice $L$ of signature $(2, n)$ can occur, and, if $n<9$, the primitive embedding $L \hookrightarrow K$ is unique up to an isometry of $K$. Similarly, if $0 \leq n^{\prime} \leq 10$, then any even integral lattice $L^{\prime}$ of signature $\left(1, n^{\prime}\right)$ can occur, and, if $n^{\prime}<10$, the embedding $L^{\prime} \hookrightarrow K$, is unique up to an isometry of $K$.

7 More precisely, let $\left(L^{\prime}\right)_{-2}$ be the set of vectors $x$ in $L^{\prime}$ with $(x, x)=-2$, and let $\mathcal{V}\left(L^{\prime}\right)^{0}$ be one component of the set of vectors $x \in L_{\mathbb{R}}^{\prime}$ with $(x, x)>0$, i.e., a choice of positive light cone. Finally, let $C\left(L^{\prime}\right)^{+}$be a component of

$$
\mathcal{V}\left(L^{\prime}\right)^{0}-\bigcup_{x \in\left(L^{\prime}\right)_{-2}} x^{\perp} .
$$

Then the ample cone condition is that

$$
\lambda_{\mathbb{R}}\left(C\left(L^{\prime}\right)^{+}\right) \cap \mathcal{K}_{X} \neq \emptyset
$$

where $\mathcal{K}_{X}$ is the closure of the ample cone in $\operatorname{Pic}(X)_{\mathbb{R}}$. 
(3) In such a family the generic element has Picard number $\rho(X)=$ $\operatorname{rank}\left(L^{\prime}\right)=20-n$,

For small values of $n$, the resulting $M_{\Gamma}$ 's are familiar classical objects. Here is a little table:

\begin{tabular}{|l|c|c|l|l|}
\hline$n$ & $\rho$ & $G=\mathrm{SO}(V)$ & $M_{\Gamma}$ classically & accidental iso. \\
\hline \hline 0 & 20 & $\mathrm{SO}(2)$ & $\mathrm{U}(1)$ & CM \\
\hline 1 & 19 & $\mathrm{SO}(2,1)$ & $\mathrm{SL}(2, \mathbb{R})$ & Shimura curves \\
\hline 2 & 18 & $\mathrm{SO}(2,2)$ & $\mathrm{SL}(2, \mathbb{R}) \times \mathrm{SL}(2, \mathbb{R})$ & Hilbert modular surfaces \\
\hline 3 & 17 & $\mathrm{SO}(2,3)$ & $\mathrm{Sp}(2, \mathbb{R})$ & Siegel 3-folds \\
\hline 4 & 16 & $\mathrm{SO}(2,4)$ & $\mathrm{SU}(2,2)$ & unitary Shimura 4-folds \\
\hline- & - & - & - & - \\
\hline 19 & 1 & $\mathrm{SO}(2,19)$ & - & moduli of polarized K3's \\
\hline
\end{tabular}

For example, for $n=19$, we have $L^{\prime}=(2 d)$, and we get the moduli space of polarized K3's of polarization degree $2 d$. Note that $K$ is an even lattice and represents every positive even integer.

At the other extreme, for $n=0$, we have $\operatorname{sig}(L)=(2,0), \operatorname{rank} L^{\prime}=20$, and $X$ is a singular K3 surface.

\subsection{Modular interpretation of the special cycles}

In the case of families of lattice polarized $\mathrm{K} 3$ surfaces $M_{\Gamma}$ for a lattice $L$ of signature $(2, n)$ as described in the previous section, vectors in $L$ correspond to additional elements of $\operatorname{Pic}(X)$. Let $N=\mathbb{Z}^{r}$, and, for $T \in \operatorname{Sym}_{r}(\mathbb{Z})_{>0}$, let $N=N_{T}$ be the quadratic lattice of signature $(0, r)$ defined by $-T$.

Proposition 3.2 The codimension $r$ cycle $Z(T)$ can be identified with the locus of objects $(X, \lambda, j)$ where

$$
j: N_{T} \hookrightarrow \operatorname{Pic}(X)
$$

is a quadratic embedding with

$$
j\left(N_{T}\right) \cdot \lambda\left(L^{\prime}\right)=0 .
$$

Here, if $(X, \lambda, u)$ is a marked object, then

$$
u \circ j: \mathbb{Z}^{r}=N_{T} \hookrightarrow L, \quad e_{i} \mapsto x_{i},
$$

determines an $r$-tuple $\mathbf{x} \in L^{r}$ with $T(\mathbf{x})=-T$ and the period point of $(X, u)$ lies in $D_{\mathbf{x}} \subset D(L)$. 
Remark 3.3 (1) In this construction, we have fixed the basis $\mathbb{Z}^{r} \simeq N$. A change in this basis corresponds to a right multiplication of the row vectors $\mathbf{x}$ by an element of $\mathrm{GL}_{r}(\mathbb{Z})$.

(2) For $r=1$, we are imposing a single additional class in $\operatorname{Pic}(X)$ and the $Z(t)$ 's are essentially the Noether-Lefschetz divisors in $M_{\Gamma}, c f$. [24].

\subsection{Some applications:}

I. Suppose that, for a smooth projective curve $C$,

$$
i_{\pi}: C \longrightarrow M_{\Gamma}
$$

is a morphism corresponding to a family

$$
\pi: X \longrightarrow C
$$

of $L^{\prime}$-polarized K3 surfaces. Recall that $Z(t) \rightarrow M_{\Gamma}$ is the locus of collections $(X, \lambda, j), j \cdot j=-t$, and consider the fiber product

$$
\begin{array}{cc}
Z(t) \times{ }_{M_{\Gamma}} C & \longrightarrow Z(t) \\
\downarrow & \downarrow \\
C & \longrightarrow M_{\Gamma} .
\end{array}
$$

Then, for $[C] \in H_{c}^{2 n-2}\left(M_{\Gamma}\right)$,

$$
\begin{aligned}
{[Z(t)] \cdot[C] } & =\left.\operatorname{deg} \mathcal{O}_{Z(t)}\right|_{C} . \\
& =\sum_{z \in C} \#\left\{\left(X_{z}, \lambda_{z}, j\right) \mid j \cdot j=-t, j \cdot \lambda=0\right\} \quad \text { (generically) } \\
& =: m(t, X / C)=\text { Noether-Lefschetz number. }
\end{aligned}
$$

For example, if the Picard number of a generic member of the family is $20-n$, then $C$ is not contained in any of the $Z(t)$ 's and the loci in question are all finite sets of points.

\section{Corollary 3.4}

$$
\phi_{1}(\tau) \cdot[C]=\left.\operatorname{deg} \mathcal{L}\right|_{C}+\sum_{t \in \mathbb{Z}_{>0}} m(t, X / C) q^{t},
$$

is an elliptic modular form of weight $\frac{n}{2}+1$ and level determined by L. In particular, the numbers $m(t, X / C)$ are the Fourier coefficients of this form. 
This result is due to Maulik-Pandharipande, 24, where it is derived from Borcherds' Theorem and its significance in Gromov-Witten theory is explained.

II. Similarly, suppose that

$$
\pi: X \longrightarrow S, \quad i_{\pi}: S \longrightarrow M_{\Gamma}
$$

is a family of $L^{\prime}$-polarized $\mathrm{K} 3$ surfaces where $S$ is a projective surface. For $T \in \operatorname{Sym}_{2}(\mathbb{Z})_{>0}, Z(T) \rightarrow M_{\Gamma}$ is the locus of collections $(X, \lambda, \mathbf{j})$, where $\mathbf{j}=\left[j_{1}, j_{2}\right]$ is a pair of classes in $\operatorname{Pic}(X)$ orthogonal to $\lambda\left(L^{\prime}\right)$ and with matrix of inner products $\mathbf{j} \cdot \mathbf{j}=-T$. Consider

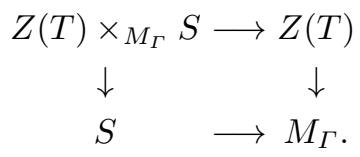

Then, for the cohomology class $[S] \in H_{c}^{2 n-4}\left(M_{\Gamma}\right)$, we can define

$$
\begin{aligned}
{[Z(T)] \cdot[S] } & =\sum_{z \in S} \#\left\{\left(X_{z}, \lambda_{z}, \mathbf{j}\right) \mid \mathbf{j} \cdot \mathbf{j}=-T, \mathbf{j} \cdot \lambda=0\right\} \quad \text { (generically) } \\
& =: m(T, X / S)=\text { a higher Noether-Lefschetz number. }
\end{aligned}
$$

Here there can be curves in $Z(T) \cap S$ and, in this case, more care must be taken to interpret the intersection number $[Z(T)] \cdot[S]$. By the modularity results above, the $m(T, X / S)$ are Fourier coefficients of a Siegel modular form of genus 2 and weight $\frac{n}{2}+1$. It would be interesting to compute these Siegel modular forms for specific families $X \rightarrow S$, e.g., ones coming from explicit classical geometry.

III. Suppose that $L$ has signature $(2,2)$ and is anisotropic. Then we have a family where $S=M_{\Gamma}$ is itself a projective surface, so that

$$
\phi_{2}\left(\tau, M_{\Gamma}\right) \in H^{4}\left(M_{\Gamma}\right) \stackrel{\text { deg }}{\longrightarrow} \mathbb{C} .
$$

The following result from [11] is obtained by combining the results of [16] with the extended Siegel-Weil formula of [17].

Theorem 3.5 Assume that condition (1) below holds. Then

$$
\operatorname{deg} \phi_{2}\left(\tau, M_{\Gamma}\right)=E\left(\tau, \frac{1}{2}, L\right)
$$

where $E(\tau, s, L)$ is a Siegel Eisenstein series of genus 2 and weight 2 associated to $L$, evaluated at the Siegel-Weil critical point $s=s_{0}=\frac{1}{2}$.

Note that, for $T>0, Z(T)$ is a 0 -cycle, and, when $T \geq 0$ has rank 1 , then $Z(T)^{\text {naive }}$ is a curve on $M_{\Gamma}$. Thus 


$$
\begin{aligned}
\operatorname{deg} \phi_{2}\left(\tau, M_{\Gamma}\right)=\operatorname{vol}\left(M_{\Gamma}, \omega^{2}\right)+\sum_{\operatorname{rank}(T)=1} \operatorname{vol}\left(Z(T)^{\text {naive }}, \omega\right) q^{T} & \\
& +\sum_{T>0} \operatorname{deg}(Z(T)) q^{T} .
\end{aligned}
$$

Here, the Siegel-Eisenstein series is defined by

$$
E(\tau, s, L)=\sum_{\gamma \in \Gamma_{\infty}^{\prime} \backslash \Gamma^{\prime}} \operatorname{det}(c \tau+d)^{\frac{n}{2}+1}|\operatorname{det}(c \tau+d)|^{s-s_{0}} \operatorname{det}(v)^{\frac{1}{2} s-s_{0}} \Phi(\gamma, L),
$$

where

$$
\gamma=\left(\begin{array}{ll}
a & b \\
c & d
\end{array}\right) \in \Gamma^{\prime}=\operatorname{Sp}_{2}(\mathbb{Z}) .
$$

and $\Phi(\gamma, L)$ is a generalized Gauss sum attached to $\gamma$ and $L$, 31, 12. This series is termwise absolutely convergent for $\operatorname{Re}(s)>\frac{3}{2}$. Its value at $s_{0}=\frac{1}{2}$ is defined by analytic continuation, [17. The main point behind Theorem 3.5 is that, as explained in Remark 2.4 (3), there is a genus 2 theta function $\theta\left(\tau, \varphi_{K M}^{(2)}\right)$ valued in $A^{(2,2)}\left(M_{\Gamma}\right)$, i.e., in top degree forms, and the degree generating series $\operatorname{deg} \phi_{2}\left(\tau, M_{\Gamma}\right)$ is obtained by integrating this form over $M_{\Gamma}$. Let $H=\mathrm{O}(V)$ be the orthogonal group of $V$ and assume that there is an open compact subgroup $K \subset H\left(\mathbb{A}_{f}\right)$, the group of finite adèle points of $H$, such that

$$
H(\mathbb{A})=H(\mathbb{Q}) H(\mathbb{R}) K, \quad \Gamma=H(\mathbb{Q}) \cap K .
$$

Then the geometric integral of $\theta\left(\tau, \varphi_{K M}^{(2)}\right)$ over

$$
M_{\Gamma} \simeq H(\mathbb{Q}) \backslash H(\mathbb{A}) / K_{\infty} K
$$

coincides with the integral of a scalar valued theta function on $H(\mathbb{A})$ over the adelic quotient $H(\mathbb{Q}) \backslash H(\mathbb{A})$ - cf. [13, section 4, for a more detailed discussion. The extended Siegel-Weil formula, [17, 12, identifies the result as a special value at $s=s_{0}$, perhaps outside the range of absolute convergence, of a certain Eisenstein series attached to $L$.

IV. Here is an amusing example. Let $F=\mathbb{Q}(\sqrt{d}), d \in \mathbb{Z}_{>0}$ square free, be a real quadratic field with ring of integers $O_{F}$. Let $M$ be a projective $O_{F}$-lattice with a symmetric $O_{F}$-bilinear form $(,)_{M}$, and suppose that the signature of $M$ is given by

$$
\operatorname{sig}(M)=((2, m),(0, m+2)) .
$$

Let $L$ be $M$, viewed as a $\mathbb{Z}$-module, with bilinear form $(,)_{L}$ given by

$$
(x, y)_{L}=\operatorname{tr}_{F / \mathbb{Q}}(x, y)_{M} .
$$

The signature of $L$ is $(2,2 m+2)$. Let $V=L \otimes_{\mathbb{Z}} \mathbb{Q}$ and note that 


$$
V \otimes_{\mathbb{Q}} \mathbb{R}=\left(V \otimes_{F} F\right) \otimes_{\mathbb{Q}} \mathbb{R}=\left(V \otimes_{F, \sigma_{1}} \mathbb{R}\right) \times\left(V \otimes_{F, \sigma_{2}} \mathbb{R}\right)
$$

where $\sigma_{1}$ and $\sigma_{2}$ are the two real embeddings of $F$. The two factors on the right have signatures $(2, m)$ and $(0, m+2)$ respectively. Then there is an embedding

$$
D(M)=D\left(V \otimes_{F, \sigma_{1}} \mathbb{R}\right) \hookrightarrow D\left(V_{\mathbb{R}}\right)=D(L) .
$$

Let $\Gamma_{M} \subset \Gamma$ be the subgroup of $O_{F}$-linear isometries of in $\Gamma$. Then

$$
\Gamma_{M} \backslash D(M) \longrightarrow \Gamma \backslash D(L)
$$

is an algebraic cycle of codimension $m+2$, and, since the quotient $\Gamma_{M} \backslash D(M)$ is compact, this cycle is projective.

For example, when $m=2$, we get a projective surface

$$
S \longrightarrow M_{\Gamma}=M_{\Gamma}(2,6), \quad[S] \in H_{c}^{8}\left(M_{\Gamma}\right) .
$$

It would be nice to have a concrete description of the corresponding family of K3 surfaces $X \rightarrow S$. The Siegel modular generating function for higher Noether-Lefschetz numbers for this family is

$$
\phi_{2}\left(\tau, M_{\Gamma}\right) \cdot[S]=i^{*} E_{F}\left(\tau, \frac{1}{2}, \Phi\right), \quad i: \mathrm{SL}_{2} / \mathbb{Q} \longrightarrow \mathrm{SL}_{2} / F
$$

the pullback of the special value of a genus 2 Hilbert-Siegel Eisenstein series $E_{F}\left(\tau, \frac{1}{2}, \Phi\right)$ of weight $(2,2)$ over $F$ to a weight 4 modular Siegel modular form over $\mathbb{Q}$ ! This is again a consequence of the extended Siegel-Weil formula together with a seesaw identity, [10. Note that, in general, such a pullback will now have lots of cuspidal components and so the Fourier coefficients of such a form are essentially more complicated than those of an Eisenstein series like that occurring in Theorem 3.5 .

Remark 3.6 A more or less explicit geometric construction of an example for $m=1$ is described in [33], section 3. In this case, $\operatorname{sig}(M)=((2,1),(0,3))$ and the base of the family will be a Shimura curve $C$ over $F$. In this case, the generating series for the Noether-Lefschetz numbers will be an elliptic modular form of weight 3 arising as the pullback for a Hilbert modular Eisenstein series of weight $(3 / 2,3 / 2)$.

V. The previous example can be further generalized. Suppose that $F$ is a totally real number field with ring of integers $O_{F},|F: \mathbb{Q}|=d>1$, and real embeddings $\sigma_{i}: F \hookrightarrow \mathbb{R}, 1 \leq i \leq d$. Let $M,(,)_{M}$ be a quadratic $O_{F}$-lattice of rank $m+2$ over $O_{F}$ and with

$$
\operatorname{sig}(M)=\left((2, m),(0, m+2)^{d-1}\right)
$$

Define a quadratic lattice $L$ as in example $\mathbf{I V}$, so that 


$$
\operatorname{sig}(L)=(2, d(m+2)-2) .
$$

Again setting $V=L \otimes_{\mathbb{Z}} \mathbb{Q}$, we have

$$
V \otimes_{\mathbb{Q}} \mathbb{R}=\left(V \otimes_{F, \sigma_{1}} \mathbb{R}\right) \times\left(V \otimes_{F, \sigma_{2}} \mathbb{R}\right) \times \cdots \times\left(V \otimes_{F, \sigma_{d}} \mathbb{R}\right),
$$

an embedding

$$
D(M)=D\left(V \otimes_{F, \sigma_{1}} \mathbb{R}\right) \hookrightarrow D\left(V_{\mathbb{R}}\right)=D(L),
$$

and a projective algebraic cycle

$$
Y=\Gamma_{M} \backslash D(M) \longrightarrow \Gamma \backslash D(L)=M_{\Gamma},
$$

of codimension $(d-1)(m+2)$ and dimension $m$. For this construction to fall into the world of K3 moduli, we must require that

$$
2 \leq d(m+2) \leq 21
$$

and hence we have the following little table of the possibilities:

(Note that $N=\operatorname{dim} M_{\Gamma}=d(m+2)-2$.)

\begin{tabular}{|c|c|c|c|c|c|c|c|c|c|}
\hline$d$ & 2 & 3 & 4 & 5 & 6 & 7 & 8 & 9 & 10 \\
\hline$m=\operatorname{dim} Y$ & $0 \leq m \leq 8$ & $0 \leq m \leq 5$ & $0 \leq m \leq 3$ & $0 \leq m \leq 2$ & 0,1 & 0,1 & 0 & 0 & 0 \\
\hline$N=\operatorname{dim} M_{\Gamma}$ & $2 \leq N \leq 18$ & $4 \leq N \leq 19$ & $6 \leq N \leq 19$ & $8 \leq N \leq 18$ & 10,16 & 12,19 & 14 & 16 & 18 \\
\hline
\end{tabular}

It would be interesting to give an account of the families of K3 surfaces over the projective varieties $Y$ occurring here.

One nice case is the following. Let $k=\mathbb{Q}\left(\zeta_{13}\right)$ be the 13 th cyclotomic field and let $F=\mathbb{Q}\left(\zeta_{13}+\zeta_{13}^{-1}\right)$, so that $|F: \mathbb{Q}|=6$. Let $M \otimes_{O_{F}} F$ to be the space of elements of trace 0 in a quaternion algebra $B / F$ with $B \otimes_{F \sigma_{1}} \mathbb{R}=M_{2}(\mathbb{R})$ and $B \otimes_{F, \sigma_{i}} \mathbb{R} \simeq \mathbb{H}$, the Hamiltonian quaternions, for $i>1$. To specify $B$, we need to choose an additional set $\Sigma_{f}(B)$ of finite places of $F$ with $\left|\Sigma_{f}(B)\right|$ odd. The algebra $B$ is determined by the condition that, for a finite place $v$ of $F, B \otimes_{F} F_{v}$ is a division algebra if and only if $v \in \Sigma_{f}(B)$. The simplest choice would be $\Sigma_{f}(B)=\left\{v_{13}\right\}$, where $v_{13}$ is the unique place above 13 . We fix a maximal order $O_{B}$ in $B$ and let $M$ be the set of trace 0 elements in it. As quadratic form on $M$, we take $(x, y)=\operatorname{tr}\left(x y^{\iota}\right)$. In this case, $V=L \otimes_{\mathbb{Z}} \mathbb{Q}$ has signature $(2,16)$, and, as noted in Remark 3.1 (1), the rational quadratic space $V$ occurs as a summand of $K_{\mathbb{Q}}$. I have not checked 8 if the lattice $L$ just defined can occur as $V \cap K$. In any case, we obtain a Shimura curve $C$ embedded in the 16-dimensional moduli space $M_{\Gamma}$, and the generating series for the Noether-Lefschetz numbers for the associated family of K3 surfaces

8 If it differs from the primitive lattice $V \cap K$, then some 'level structure' will have to be introduced. 
will be an elliptic modular form of weight 9 arising as the pullback of a Hilbert modular Eisenstein series of weight $(3 / 2, \ldots, 3 / 2)$ for $F$.

\section{Kuga-Satake abelian varieties and special endomorphisms}

In this last section, we give another interpretation of the special cycles $Z(T)$. For convenience, we change the sign and consider rational quadratic forms of signature $(n, 2)$.

\subsection{The Kuga-Satake construction}

The moduli spaces $M_{\Gamma}$ of lattice polarized K3 surfaces carry families of abelian varieties arising from (a slight variant of) the Kuga-Satake construction, which we now review.

For a quadratic lattice $L$ of signature $(n, 2)$ and for $V=L_{\mathbb{Q}}$, let

$$
C=C(V)=C^{+}(V) \oplus C^{-}(V)
$$

be the Clifford algebra of $V$. For a basis $v_{1}, \ldots, v_{n+2}$ of $V$, let $\delta=v_{1} \cdots v_{n+2} \in$ $C(V)$, and let $k=\mathbb{Q}(\delta)$ be the discriminant field. For $n$ even, $\delta \in C^{+}(V), k$ is the center of $C^{+}(V)$, and $\delta$ anticommutes with elements of $C^{-}(V)$. For $n$ is odd, $\delta \in C^{-}(V)$ and $k$ is central in all of $C(V)$. Now

$$
O_{C}=C(L)=\text { Clifford algebra of } L
$$

is an order in $C$ and $O_{k}=k \cap C(L)$ is an order in $k$. We obtain a real torus

$$
A^{\text {top }}=C\left(L_{\mathbb{R}}\right) / C(L)
$$

of dimension $2^{n+2}$ with an action

$$
\iota: C(L) \otimes_{\mathbb{Z}} O_{k} \longrightarrow \operatorname{End}\left(A^{\text {top }}\right), \quad \iota(c \otimes \alpha): x \longmapsto c x \alpha,
$$

via left-right multiplication. Let

$$
G=\operatorname{GSpin}(V)=\left\{g \in C^{+}(V)^{\times} \mid g V g^{-1}=V\right\}
$$

and, for an element $a \in C^{+}(L) \cap C(V)^{\times}$with $a^{\iota}=-a$, define an alternating form on $C$ by

$$
\langle x, y\rangle=\langle x, y\rangle_{a}=\operatorname{tr}\left(a x y^{\iota}\right) .
$$


Here $x \mapsto x^{\iota}$ is the main involution on $C(V)$, determined by the conditions $(x y)^{\iota}=y^{\iota} x^{\iota}$ for all $x, y \in C$, and $v^{\iota}=v$ for all $v \in V \subset C^{-}(V)$. Note that for $g \in G$,

$$
\langle x g, y g\rangle=\nu(g)\langle x, y\rangle, \quad \nu(g)=g g^{\iota}=\text { spinor norm. }
$$

A variation of complex structures on $A^{\text {top }}$ is defined as follows. For an oriented negative 2-plane $z \in D(L)$ in $V_{\mathbb{R}}$, let $z_{1}, z_{2}$ be a properly oriented orthonormal basis and let $j_{z}=z_{1} z_{2} \in C^{+}\left(V_{\mathbb{R}}\right)$. This element is independent of the choice of basis. Note that $j_{z}^{2}=-1$, so that right multiplication by $j_{z}$ defines a complex structure on $C\left(V_{\mathbb{R}}\right)$, and hence we have a complex torus $A_{z}=\left(A^{\text {top }}, j_{z}\right)$ for each $z \in D(L)$. Since the action of $C(L) \otimes O_{k}$ commutes with the right multiplication by $j_{z}$, we have

$$
\iota: O_{C} \otimes_{\mathbb{Z}} O_{k} \longrightarrow \operatorname{End}\left(A_{z}\right)
$$

with

$$
\iota(c \otimes \alpha)^{*}=\iota\left(c^{*} \otimes \alpha^{\iota}\right), \quad c^{*}=a c^{\iota} a^{-1},
$$

for the Rosati involution determined by $\langle$, $\rangle$. If $\gamma \in \Gamma \subset \Gamma_{L}$, and 9 if $\tilde{\gamma} \in O_{C}^{\times}$ is an element mapping to $\gamma$ under the natural homomorphism GSpin $(\mathrm{V}) \rightarrow$ $\mathrm{SO}(V)$, then right multiplication by $\tilde{\gamma}$ on $C\left(L_{\mathbb{R}}\right)$ induces an isomorphism of the complex tori $A_{\gamma(z)}$ and $A_{z}$, equivariant for the action of $O_{C} \otimes O_{k}$. Finally, fix a rational splitting $V=V^{+}+V^{-}$of signature $(n, 0)+(0,2)$ and let $a_{1}$, $a_{2}$ be a $\mathbb{Z}$-basis for the negative definite lattice $L^{-}=L \cap V^{-}$. Let $a=a_{1} a_{2}$. With this choice of $a$, the form $\langle,\rangle_{a}$ is $\mathbb{Z}$-valued on $C(L)$ and defines a Riemann form on each complex torus $A_{z}$, i.e., the form $\left\langle x j_{z}, y\right\rangle_{a}$ is symmetric and definite (hence positive definite on one of the connected components of $D(L)$ ). Thus, $M_{\Gamma}$ carries a family of polarized abelian varieties:

$$
\pi: \operatorname{KS}(L) \longrightarrow M_{\Gamma}, \quad\left(A_{z}, \iota, \lambda\right), \quad \operatorname{dim} A_{z}=2^{n+1}
$$

with endomorphisms (11). Note that these abelian varieties are $\mathbb{Z}_{2}$-graded,

$$
A_{z}=A_{z}^{+} \oplus A_{z}^{-}
$$

since the construction respects the decomposition $C(L)=C^{+}(L) \oplus C^{-}(L)$.

Remark 4.1 (1) For $n \leq 4, M_{\Gamma}$ is the moduli space for such PE type abelian varieties and is a Shimura variety of PEL typ 10 .

(2) For general $n \geq 5$, the abelian varieties in the family are not characterized by the given PE; more Hodge classes are required, and $M_{\Gamma}$ is a Shimura variety of Hodge type. The family $\pi$ corresponds to a morphism

${ }^{9}$ In general, there is an orbifold issue here that can be eliminated by introducing a suitable level structure.

10 We could add a level structure here. 


$$
i_{\pi}: M_{\Gamma} \longrightarrow A_{g}, \quad g=2^{n+1} .
$$

\subsection{Special cycles and special endomorphisms}

Special endomorphisms arise as follows. For $x \in L$, let

$$
r_{x}: A^{\text {top }} \longrightarrow A^{\text {top }}=C\left(L_{\mathbb{R}}\right) / C(L),
$$

be the endomorphism induced by right multiplication by $x$. Note that this endomorphism has degree 1 with respect to the $\mathbb{Z}_{2}$-grading, and that, since $x^{2}=Q(x)$ in $C(L)$,

$$
r_{x}^{2}=[Q(x)]
$$

is just multiplication by the integer $Q(x)=(x, x) \in \mathbb{Z}$. Moreover,

$$
r_{x} \circ \iota(c \otimes b)= \begin{cases}\iota(c \otimes b) \circ r_{x} & \text { for } n \text { odd } \\ \iota\left(c \otimes b^{\sigma}\right) \circ r_{x} & \text { for } n \text { even. }\end{cases}
$$

and the adjoint of $r_{x}$ with respect to $\langle$,$\rangle is$

$$
\left(r_{x}\right)^{*}=r_{x^{\iota}}=r_{x} .
$$

Definition 4.2 For a given $z \in D(L)$, the endomorphism $r_{x}$ is said to be a special endomorphism of the abelian variety $A_{z}$ when it is holomorphic, i.e., when $r_{x} \in \operatorname{End}\left(A_{z}\right)$.

Lemma 4.3 Let $L\left(A_{z}\right)$ be the space of special endomorphisms of $A_{z}$, with quadratic form defined by $j^{2}=Q(j)$. Then

$$
L\left(A_{z}\right)=L \cap z^{\perp}
$$

is an isometry.

Note that, $r_{x}$ is a special endomorphism of $A_{z}$ precisely when $x$ commutes with $j_{z}$. Since conjugation by $j_{z}$ induces the endomorphism of $V$ that is -1 on $z$ and +1 on $z^{\perp}$, it follows that $r_{x}$ is a special endomorphism precisely when $x \in z^{\perp}$, i.e., $z \in D_{x}$.

Corollary 4.4 For $T \in \operatorname{Sym}_{r}(\mathbb{Z})_{>0}$, the special cycle $Z(T)$ is the image in $M_{\Gamma}$ of

$$
\tilde{Z}(T)=\left\{\left(A_{z}, \mathbf{j}\right) \mid \mathbf{j} \in L\left(A_{z}\right)^{r},(\mathbf{j}, \mathbf{j})=T\right\} .
$$


In fact, to give the right definition of special endomorphism, one should start with an object $(A, \iota, \lambda, \epsilon, \xi)$ where $A$ is an abelian variety (scheme) with $O_{C} \otimes O_{k}$-action $\iota$, a $\mathbb{Z} / 2 \mathbb{Z}$-grading $\epsilon$ and additional Hodge tensors $\xi$. A special endomorphism is then an element $j \in \operatorname{End}(A)$ of degree 1 with respect to the $\mathbb{Z} / 2 \mathbb{Z}$-grading satisfying conditions (11) and (2), with an additional compatibility with respect to the Hodge tensors $\xi$. For $0 \leq n \leq 3$, where the classes $\xi$ can be expressed in terms of endomorphisms, such a definition of special endomorphism was given in [20] $(n=0), 21](n=1),[18,(n=2)$, and 19] $(n=3)$ and used to define special cycles in integral models of the associated PEL type Shimura varieties. The results of these papers concerning the intersection number of integral special cycles and Fourier coefficients of

modular forms should have some consequences in the arithmetic theory of K3 surfaces. To reveal it one would have to relate these integral models to the integral models of moduli spaces of K3 surfaces developed by Rizov, 27, 28]. The extension to the case of general $n$, i.e., to integral models of Shimura varieties attached to $\operatorname{GSpin}(n, 2)$ is the subject of ongoing work of Andreatta-Goren, [1] and of Howard-Madapusi, 8].

Remark 4.5 In the references that follow, I have made no attempt at completeness and apologize in advance for the many omitted citations.

\section{References}

1. F. Andreatta and E. Goren, Research report on a conjecture of Bruinier-Yang, Oberwolfach report, July 2012.

2. R. E. Borcherds, The Gross-Kohnen-Zagier theorem in higher dimensions, Duke Math. J. 97 (1999), 219-233.

3. I. Dolgachev, Mirror symmetry for lattice polarized K3 surfaces, J. Math. Sci. 81 (1996), 2599-2630.

4. I. Dolgachev and S. Kondo, Moduli spaces of K3 surfaces and complex ball quotients, in Arithmetic and geometry around hypergeometric functions, Progr. Math. 260, Birkhäuser, Basel, 2007, pp. 43-100.

5. J. Funke and J. Millson, Cycles in hyperbolic manifolds of non-compact type and Fourier coefficients of Siegel modular forms, Manuscripta Math. 107 (2002), 409-444.

6. Cycles with local coefficients for orthogonal groups and vector valued Siegel modular forms, Amer. J. Math. 128 (2006), 899-948.

7. Spectacle cycles with coefficients and modular forms of half-integral weight, Adv. Lect. in Math. (ALM) 19, Int. Press, Somerville, MA, 2011.

8. B. Howard and K. Madapusi, private communication.

9. A. Klemm, D. Maulik, R. Pandharipande, and E. Scheidegger, Noether-Lefschetz theory and the Yau-Zaslow conjecture, Jour. of the AMS, 23 (2010), 1013-1040.

10. S. Kudla, Seesaw dual reductive pairs, in Automorphic Forms of Several Variables, Taniguchi Symposium, Katata 1983, Progr. Math. 46, Birkhäuser, Boston, 1984, pp. $244-268$.

11. Algebraic cycles on Shimura varieties of orthogonal type, Duke Math. J. 86 (1997), no. 1, 39-78.

12. Applications, W.-T. Gan, S. Kudla and Y. Tschinkel, eds, Progress in Math. 258, Birkhäuser, Boston, 2008. 
13. Integrals of Borcherds forms, Compositio Math., 137, (2003), 293-349.

14. S. Kudla and J. Millson, The theta correspondence and harmonic forms I, Math. Annalen, 274 (1986), 353-378.

15. The theta correspondence and harmonic forms II, Math. Annalen, 277 (1987), $267-314$.

16. Intersection numbers of cycles on locally symmetric spaces and Fourier coefficients of holomorphic modular forms in several complex variables, Publ. Math. IHES, 71 (1990), 121-172.

17. S. Kudla and S. Rallis, On the Weil-Siegel formula, Crelle 387, (1988) 1-68.

18. S. Kudla and M. Rapoport, Arithmetic Hirzebruch-Zagier cycles, J. reine angew. Math., 515 (1999), 155-244.

19. Cycles on Siegel 3-folds and derivatives of Eisenstein series, Ann. sci. École. Norm. Sup., 33 (2000), 695-756.

20. S. Kudla, M. Rapoport and T. Yang, On the derivative of an Eisenstein series of weight 1, Internat. Math. Res. Notices 7 (1999), 347-385.

21. Modular Forms and Special Cycles on Shimura Curves, Annals of Math. Studies, 161, Princeton University Press, Princeton, 2006.

22. M. Kuga and I. Satake, Abelian varieties attached to polarized K3 surfaces, Math. Annalen 109, (1967), 239-242.

23. K. Madapusi, Regular integral models of Spin Shimura varieties, in preparation.

24. D. Maulik and R. Pandharipande Gromov-Witten theory and Noether-Lefschetz theory, arXiv:0705.1653v2, 2010.

25. D. Morrison, On K3 surfaces with large Picard number, Invent. Math. 75 (1984), $105-121$.

26. V. Nikulin, Integral symmetric bilinear forms and some of their applications, Izv. Akad. Nauk. SSSR 43 (1979), 111-177; Maht. USSR Izvestija 14 (1980), 103-167.

27. J. Rizov, Moduli stacks of polarized K3 surfaces in mixed characteristic, Serdica Math. J., 32 (2006), 131-178.

28. Kuga-Satake abelian varieties in positive characteristic, J.Reine Angew. Math. 648 (2010), 13-67.

29. I. Satake, Clifford algebras and families of abelian varieties, Nagoya Math. J., 27 (1966), 435-446.

30. G. Shimura, On modular forms of half-integral weight, Annals of Math. (2) 97 (1973), 440-481.

31. C. L. Siegel, Lecture on the Analytic Theory of Quadratic Forms, Buchhandlung Robert Peppmüller, Göttingen, Germany, 1963

32. B. van Geemen, Kuga-Satake varieties and the Hodge conjecture, in The Arithmetic and Geometry of Algebraic Cycles, eds BB. Gordon et. al., pp 51-82, Kluwer Acad. Publ. 2000.

33. Real multiplication on K3 surfaces and Kuga Satake varieties Michigan Math. J. 56 (2008), 375-399.

34. Wei Zhang, Modularity of generating functions of special cycles on Shimura varieties, Ph.D. thesis, Columbia University, 2009. 